\title{
Artigo Original / Original Paper \\ Chave interativa de espécies arbóreas em florestas estacionais do sudoeste da Bahia
}

\author{
Interactive key of arboreal species in seasonal forests of Southwest of Bahia
}

\author{
Inkamauta Valeda Cerda Plazas ${ }^{1,2,3}$ \& Alessandro de Paula ${ }^{1}$
}

\begin{abstract}
Resumo
As florestas estacionais são as fitofísionomias do bioma Mata Atlântica mais ameaçadas, principalmente, pela pressão do agronegócio e pelo processo de urbanização. Diante desse cenário, o conhecimento de sua estrutura e, principalmente, de sua composição florística, são fundamentais para os processos de conservação e preservação dos recursos naturais. No intuito de auxiliar o processo de identificação de espécies, este trabalho teve como objetivo confeccionar uma chave interativa de espécies arbóreas localizadas em florestas estacionais do sudoeste da Bahia. Na confecção da chave foram excluídas as espécies que apresentaram densidade menor ou igual a um indivíduo por hectare nos respectivos levantamentos. Para elaboração da chave dendrológica foi necessário um levantamento de caracteres morfológicos de folhas e ramos, levando em consideração suas variações, assim como sua presença ou ausência. Os dados obtidos foram lançados na plataforma "on line" do Xper ${ }^{3}$. A consistência da base de dados foi analisada por meio da ferramenta “checkbase”. Foram utilizadas 177 espécies, sendo 62 da família Leguminosae. A chave está disponível na plataforma do Xper ${ }^{3}$ com acesso livre, mostrando-se eficiente e de uso simples. A chave contempla espécies frequentes em diversos levantamentos realizados na Bahia e, ainda, pode ser utilizada para florestas estacionais em outros estados.
\end{abstract}

Palavras-chave: composição florística, dendrologia, Mata Atlântica, Xper.

\begin{abstract}
The seasonal forests are phytophysiognomies of the Atlantic Forest biology most threatened, mainly, by the pressure exerted by agribusiness pressure and the process urbanization process. In this scenario, the knowledge of its structure and, mainly, of its floristic composition, are fundamental for the processes of conservation and preservation of natural resources. In order to support the process of species identification, this work had as objective to make an interactive key of tree species located in seasonal forests of the Southwest of Bahia. In the making of the key were excluded the species with less importance and equal to one individual per hectare in the respective surveys. For the preparation of the dendrological key it was necessary a survey of the morphological characteristics of the leaf and the thin branche, taking into account its variations, as well it is presence or default. The data was released on the Xper ${ }^{3}$ online platform. The consistency of the database was analyzed through the tool "checkbase". A total of 177 species were used, 62 of the Leguminosae family. The key is available on the $\mathrm{Xper}^{3}$ platform for free access, it is efficient and simple to use. The key contemplates any species in several surveys carried out in Bahia and, can also be used for Seasonal Forests in other States.
\end{abstract}

Key words: floristic composition, dendrology, Atlantic Forest,Xper.

\footnotetext{
${ }^{1}$ Universidade Estadual do Sudoeste da Bahia - UESB, Estrada do Bem Querer km 4, s/n, Bairro Universitário, 45083-900, Vitória da Conquista, BA, Brasil.

${ }^{2}$ ORCID: < https://orcid.org/0000-0002-3204-2876>

${ }^{3}$ Autor para correspondência: inkamauta@hotmail.com
} 


\section{Introdução}

O avanço da antropização sobre áreas nativas, tem gerado uma modificação significativa no uso do solo nas diversas regiões do Brasil. Segundo a SOS Mata Atlântica \& INPE (2019), a Mata Atlântica na Bahia ocupava apenas $11,1 \%$ da sua área original em 2018. Este cenário também foi destacado por Campanili \& Prochnow (2006), quando afirmaram que no sudoeste da Bahia os ambientes naturais encontravam-se fortemente ameaçados pela expansão do agronegócio e pelo processo de urbanização próximo aos fragmentos vegetacionais.

Neste contexto estão as florestas estacionais, que são caracterizadas como fisionomias florestais que ocorrem em regiões com estações bem definidas, sendo uma seca e outra chuvosa. Estas são subdivididas em Floresta Estacional Decidual, quando apresenta perda superior a $50 \%$ de suas folhas na estação seca e Floresta Estacional Semidecidual, quando a deciduidade das folhas varia de 20 a $50 \%$ (IBGE 2012).

Diante do exposto, é fundamental o aprofundamento do conhecimento dos recursos naturais existentes. De acordo com Marchiori (2004), a partir do conhecimento é que surge a importância e a melhor forma de manejar e preservar os recursos existentes. Assim, antes da análise da estrutura, da dinâmica e do futuro manejo, é necessário a identificação das espécies (Ramalho 1978; Marchiori 2004; Pinheiro \& Almeida 2008).

Segundo Roderjan (1983), a identificação de espécies florestais pode ocorrer por três métodos: Botânica Sistemática, Anatomia da Madeira e Dendrologia, ou ainda, pela junção dos três. Pinheiro \& Almeida (2008) destacaram que a Botânica Sistemática e a Anatomia da Madeira, apesar de muito precisas, apresentam uma série de limitações, como a obtenção das partes reprodutivas ou utilização de equipamentos sofisticados. Já por meio da Dendrologia consegue-se reconhecer as plantas a nível de espécie, utilizando-se apenas caracteres morfológicos vegetativos, desde que haja um amplo conhecimento botânico. Além disso, esta operação pode ser realizada in loco não dependendo de laboratório ou equipamentos sofisticados (Ramalho 1978; Marchiori 2004; Pinheiro \& Almeida 2008).

Uma ferramenta que pode ser utilizada para facilitar o reconhecimento das espécies vegetais é a chave de identificação, que apresenta uma série de passos a serem seguidos baseados em caracteres morfológicos (Judd et al. 2009). As chaves de identificação podem ser dicotômicas ou interativas, podendo estas, serem compostas por caracteres morfológicos reprodutivos, anatômicos ou dendrológicos, ou ainda, a junção destes. A chave interativa é um programa computacional no qual o usuário entra com os caracteres e os estados de caracteres das espécies. O programa elimina táxons cujos atributos não coincidem com os apresentados pela amostra, e o processo é continuado até que apenas um táxon permaneça (Bittrich et al. 2012; Santo et al. 2013; ChrétiennotDinet et al. 2014).

Santo et al. (2013) elencaram vantagens apresentadas pelas chaves interativas, quando comparadas com as dicotômicas, como por exemplo, o uso irrestrito e livre escolha de caracteres, sendo que qualquer caractere pode ser usado e em qualquer ordem. Outro ponto relevante é a eliminação e mudança de caracteres. Também, durante o processo de produção da chave, pode-se alterar e/ou eliminar caracteres que não se encaixem devidamente às amostras que serão identificadas (Saueressig et al. 2009; Bittrich et al. 2012; Chrétiennot-Dinet et al. 2014; Carmo \& Simões 2017).

A maioria dos trabalhos sobre chaves interativas para identificação de espécies vegetais, em sua imensa maioria, utilizaram caracteres reprodutivos (Bittrich et al. 2012; Santo et al. 2013; Chrétiennot-Dinet et al. 2014; Carmo \& Simões 2017). Porém, vale destacar os trabalhos de Saueressig et al. (2009) e Eltink et al. (2011) que desenvolveram chaves interativas utilizando apenas caracteres vegetativos para Floresta Ombrófila nos municípios de Irati (PR) e Ubatuba (SP), respectivamente.

Este tipo de estudo deve ter, prioritariamente, uma abrangência regional, já que utiliza caracteres vegetativos e estes órgãos apresentam variação em função do ambiente (plasticidade ecológica), promovendo assim, uma menor precisão na identificação, gerando uma necessidade de regionalizar os estudos na área (Bradshaw 1965).

Diante do exposto, este trabalho objetivou analisar as características vegetativas para montar uma chave interativa com espécies arbóreas encontradas em Florestas Estacionais do Sudoeste da Bahia. 


\section{Material e Métodos}

As espécies utilizadas neste trabalho foram selecionadas dentre as amostradas nos levantamentos realizados pelo laboratório de Ecologia e Proteção Florestal da UESB em Florestas Estacionais da Bahia e o trabalho de Soares Filho (2012), que estudou, dentre outras, a fitogeografia e estrutura das florestas estacionais deciduais da região.

Foi gerada uma lista das espécies arbóreas de acordo com o sistema de classificação APG IV (2016). Retiraram-se os sinônimos e nomes desatualizados, segundo BFG (2018) e por meio do site The International Plant Names Index (<https:// www.ipni.org $>$ ). A partir dessa lista foram excluídas as espécies que apareceram somente em um levantamento e aquelas que se apresentavam como raras, ou seja, com densidade menor ou igual a um indivíduo por hectare (Tab. 1).

Os caracteres utilizados foram: folha (tipo, filotaxia, característica do pecíolo, presença e tipo de estípula, forma do limbo, ápice do limbo, margem do limbo, base do limbo, nervação, consistência, cor, característica da raque, pubescência, odor, presença glândulas), ramo (morfologia e coloração) e presença ou ausência

Tabela 1 - Espécies, organizadas por família, listadas nos levantamentos das florestas estacionais da Bahia, raras ou presente em apenas um levantamento.

Table 1 - Species, organized by family, listed in surveys of the Bahia seasonal forests, rare or present in only one survey.

\begin{tabular}{|c|c|}
\hline Família & Espécie \\
\hline Annonaceae & Annona crassiflora Mart. \\
\hline \multirow[t]{3}{*}{ Apocynaceae } & Aspidosperma parvifolium A.DC. \\
\hline & Himatanthus articulatus (Vahl) Woodson \\
\hline & Tabernaemontana salzmannii A.DC. \\
\hline Asteraceae & Moquiniastrum polymorphum (Less.) G. Sancho \\
\hline Capparaceae & Monilicarpa brasiliana (Banks ex DC.) Cornejo \& Iltis \\
\hline Celastraceae & Monteverdia distichophylla (Mart. ex Reissek) Biral \\
\hline Combretaceae & Terminalia actinophylla Mart. \\
\hline Erythroxylaceae & Erythroxylum caatingae Plowman \\
\hline \multirow[t]{4}{*}{ Fabaceae } & Anadenanthera colubrina var. cebil (Griseb.) Altschul \\
\hline & Cassia ferruginea (Schrad.) DC. \\
\hline & Dalbergia frutescens (Vell.) Britton \\
\hline & Machaerium amplum Benth. \\
\hline Meliaceae & Trichilia silvatica C.DC. \\
\hline Myrtaceae & Myrcia guianensis (Aubl.) DC. \\
\hline Nyctaginaceae & Guapira noxia (Netto) Lundell \\
\hline Polygonaceae & Coccoloba schwackeana Lindau \\
\hline \multirow[t]{2}{*}{ Rutaceae } & Balfourodendron molle (Miq.) Pirani \\
\hline & Hortia brasiliana Vand. ex DC. \\
\hline \multirow[t]{2}{*}{ Salicaceae } & Casearia aculeata Jacq. \\
\hline & Casearia obliqua Spreng. \\
\hline Sapindaceae & Matayba heterophylla (Mart.) Radlk. \\
\hline Urticaceae & Pourouma guianensis Aubl. \\
\hline Vochysiaceae & Vochysia thyrsoidea Pohl \\
\hline
\end{tabular}


de outros caracteres (látex e outras exsudações, embira, lenticelas, espinhos ou acúleos).

$\mathrm{Na}$ chave os descritores corresponderam aos caracteres nos quais cada espécie foi classificada, existindo caracteres dependentes, que somente podem ser a utilizados mediante a confirmação de outro caractere, por exemplo: "formato da raque" depende do caractere "composição do limbo". Já os estados do caractere, representaram as possibilidades do caractere ("simples" e "composto" são estados do caractere "limbo foliar").

A análise destes caracteres ocorreu perante amostras das espécies herborizadas (exsicatas) disponíveis no Laboratório de Ecologia e Proteção Florestal, no Herbário da Universidade Estadual do Sudoeste da Bahia (HUESBVC) e do Herbário da Universidade Estadual de Feira de Santana (HUEFS). No caso de caracteres odor e coloração do limbo, a análise teve como critério classificar as espécies conforme observado nas próprias exsicatas. Para a presença de exsudatos (látex ou outros), foram procuradas pistas da ocorrência destes por meio de sinais, como o enrugamento dos tecidos no pecíolo e ramos mais jovens.

Na plataforma do Xper ${ }^{3}$ foram inseridas todas as espécies selecionadas na aba "items" (Fig. 1). Em seguida foram adicionados os caracteres na aba "descriptive model" (Fig. 2). Por fim, na aba "Description" (Fig. 3), foram associadas às espécies os caracteres utilizados para descrição.

As figuras esquemáticas utilizadas para ilustrar os caracteres foram geradas por meio do software CorelDraw X6 para cada caractere e para cada estado de caractere. Já as figuras utilizadas para ilustrar as espécies na chave interativa dentro da plataforma foram obtidas no herbário virtual do speciesLink (<http://inct.splink.org.br $>)$. Os

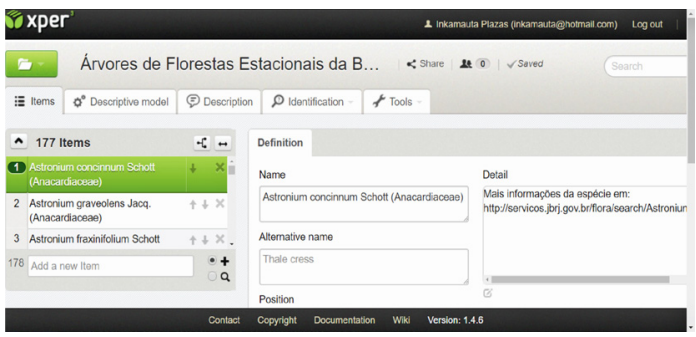

Figura 1 - Tela de entrada de dados na plataforma do $\mathrm{Xper}^{3}$ na aba de inserção de itens (táxons).

Figure 1 - Data entry screen on the Xper ${ }^{3}$ platform in the tab item insertion (taxa).

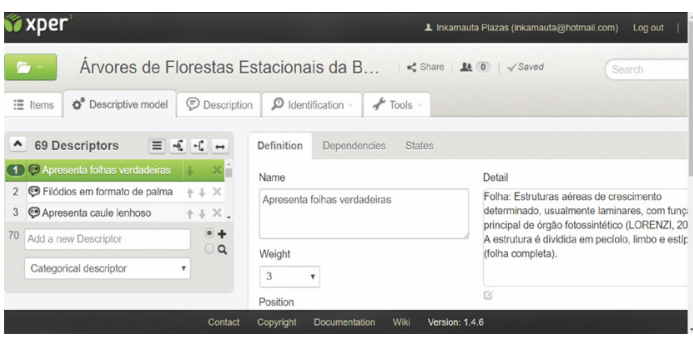

Figura 2 - Tela de entrada de dados na plataforma do Xper $^{3}$ na aba de inserção de dados dos descritores e estados.

Figure 2 - Data entry screen on the Xper ${ }^{3}$ platform in the tab insertion of descriptors and states.

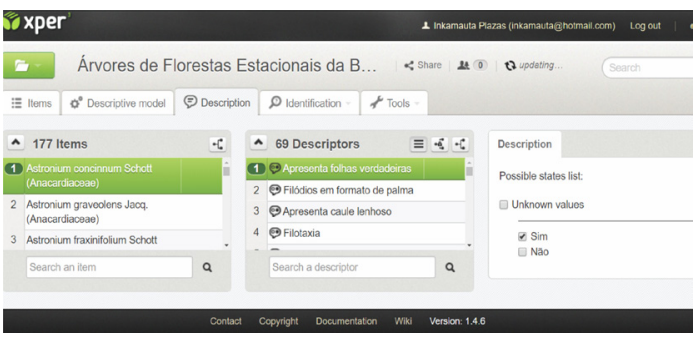

Figura 3 - Tela de entrada de dados na plataforma do $\mathrm{Xper}^{3}$ na aba de associação de itens aos descritores.

Figure 3 - Data entry screen on the Xper ${ }^{3}$ platform in the tab of the association of items to the descriptors.

conceitos morfológicos dos termos utilizados na chave foram retirados de Gonçalves \& Lorenzi (2007) e Font Quer (2001).

A consistência da base de dados foi analisada por meio da ferramenta "checkbase" verificando se espécies diferentes apresentavam os mesmos passos. Erros relacionados com espécie sem caráter preenchido ou caráter inserido de forma equivocada, também foram verificadas por meio da ferramenta "checkbase".

\section{Resultados e Discussão}

No total, 176 espécies arbóreas foram incluídas na chave interativa (Tab. 2). Para permitir a distinção das espécies, foram selecionados 68 descritores (Tab. 3), sendo 66 dependentes e 250 estados de caractere.

A família com maior riqueza foi Leguminosae (Fabaceae) com 62 espécies (Caesalpinoideae com 37 e Papilinoideae com 25 espécies), correspondendo $35 \%$ de todas as espécies da chave. Leguminosa e também foi a família mais rica nos trabalhos de Funch et al. (2008), Cardoso et 
Tabela 2 - Espécies organizadas por família utilizadas para construir a chave interativa.

Table 2 - Species organized by the family to construct an interactive key.

\begin{tabular}{|c|c|}
\hline Família & Nome científico \\
\hline \multirow[t]{7}{*}{ Anacardiaceae } & Astronium concinnum Schott \\
\hline & Astronium graveolens Jacq. \\
\hline & Astronium fraxinifolium Schott \\
\hline & Schinopsis brasiliensis Engl. \\
\hline & Spondias dulcis Parkinson \\
\hline & Tapirira guianensis Aubl. \\
\hline & Tapirira obtusa (Benth.) J.D.Mitch. \\
\hline \multirow[t]{4}{*}{ Annonaceae } & Annona sylvatica A.St.-Hil. \\
\hline & Duguetia marcgraviana Mart. \\
\hline & Hornschuchia lianarum D.M.Johnson \\
\hline & Xylopia brasiliensis Spreng. \\
\hline \multirow[t]{5}{*}{ Apocynaceae } & Aspidosperma discolor A.DC. \\
\hline & Aspidosperma polyneuron Müll.Arg. \\
\hline & Aspidosperma tomentosum Mart. \\
\hline & Geissospermum laeve Baill. \\
\hline & Tabernaemontana solanifolia A.DC. \\
\hline Araliaceae & Schefflera morototoni (Aubl.) Maguire, Steyerm. \& Frodin \\
\hline Arecaceae & Syagrus coronata Becc. \\
\hline \multirow[t]{2}{*}{ Asteraceae } & Piptocarpha axillaris (Less.) Baker \\
\hline & Piptocarpha rotundifolia Baker \\
\hline \multirow[t]{4}{*}{ Bignoniaceae } & Anemopaegma arvense (Vell.) Stellfeld ex de Souza \\
\hline & Handroanthus chrysotrichus (Mart. ex DC.) Mattos \\
\hline & Handroanthus impetiginosus (Mart. ex DC.) Mattos \\
\hline & Jacaranda caroba DC. \\
\hline \multirow[t]{6}{*}{ Boraginaceae } & Cordia americana (L.) Gottschling \& J.S.Mill. \\
\hline & Cordia glabrata A.DC. \\
\hline & Cordia incognita Gottschling \& J.S.Mill. \\
\hline & Cordia oncocalyx Allemão \\
\hline & Cordia superba Cham. \\
\hline & Varronia curassavica Jacq. \\
\hline \multirow[t]{3}{*}{ Cactaceae } & Brasiliopuntia brasiliensis A.Berger \\
\hline & Pereskia grandifolia Haw. \\
\hline & Pilosocereus pentaedrophorus (Labour.) Byles \& G.D.Rowley \\
\hline \multirow[t]{2}{*}{ Capparaceae } & Colicodendron yco Mart. \\
\hline & Monilicarpa brasiliana (DC.) Cornejo \& Iltis \\
\hline Caricaceae & Jacaratia spinosa (Aubl.) A.DC. \\
\hline \multirow[t]{2}{*}{ Caryocaraceae } & Caryocar brasiliense Cambess. \\
\hline & Caryocar edule Casar. \\
\hline \multirow[t]{3}{*}{ Celastraceae } & Maytenus gonoclada Mart. \\
\hline & Maytenus obtusifolia Mart. \\
\hline & Monteverdia rigida (Mart.) Biral \\
\hline Chrysobalanaceae & Hirtella ciliata Mart. \& Zucc. \\
\hline
\end{tabular}




\begin{tabular}{|c|c|}
\hline Família & Nome científico \\
\hline & Hirtella hebeclada Moric. \\
\hline & Licania kunthiana Hook.f. \\
\hline Cleomaceae & Melidiscus giganteus (L.) Raf. \\
\hline \multirow[t]{4}{*}{ Combretaceae } & Combretum leprosum Mart. \\
\hline & Terminalia argentea Mart. \\
\hline & Terminalia fagifolia Mart. \\
\hline & Terminalia glabrescens Mart. \\
\hline \multirow[t]{2}{*}{ Erythroxylaceae } & Erythroxylum daphnites Mart. \\
\hline & Erythroxylum polygonoides Mart. \\
\hline \multirow[t]{8}{*}{ Euphobiaceae } & Alchornea triplinervia Müll.Arg. \\
\hline & Croton agrophilus Müll.Arg. \\
\hline & Croton floribundus Spreng. \\
\hline & Croton piptocalyx Müll.Arg. \\
\hline & Maprounea guianensis Aubl. \\
\hline & Pachystroma longifolium I.M.Johnst. \\
\hline & Pleradenophora membranifolia (Müll.Arg.) Esser \& A.L.Melo \\
\hline & Sebastiania brasiliensis Spreng. \\
\hline \multirow{16}{*}{$\begin{array}{l}\text { Fabaceae (Leguminosae) - } \\
\text { Ceasalpiniodeae }\end{array}$} & Abarema jupunba (Willd.) Britton \& Killip \\
\hline & Acosmium lentiscifolium Schott \\
\hline & $\begin{array}{l}\text { Albizia inundata (Mart.) Barneby \& J.W.Grimes } \\
\text { Albizia polycephala (Benth.) Killip } \\
\text { Anadenanthera colubrina (Vell.) Brenan } \\
\text { Anadenanthera peregrina (L.) Speg. } \\
\text { Apuleia leiocarpa (Vogel) J.F.Macbr.. }\end{array}$ \\
\hline & $\begin{array}{l}\text { Chloroleucon foliolosum (Benth.) G.P.Lewis } \\
\text { Copaifera langsdorffii Desf. }\end{array}$ \\
\hline & Copaifera officinalis L. \\
\hline & $\begin{array}{l}\text { Dimorphandra jorgei M.F.Silva } \\
\text { Enterolobium contortisiliquum (Vell.) Morong } \\
\text { Goniorrhachis marginata Taub. }\end{array}$ \\
\hline & Hymenaea courbaril L. \\
\hline & Hymenaea stigonocarpa Mart. ex Hayne \\
\hline & $\begin{array}{l}\text { Inga alba Willd. } \\
\text { Inga edulis Mart. } \\
\text { Libidibia ferrea (Mart. ex Tul.) L.P.Queiroz }\end{array}$ \\
\hline & $\begin{array}{l}\text { Parapiptadenia rigida (Benth.) Brenan } \\
\text { Peltogyne angustiflora Ducke }\end{array}$ \\
\hline & Peltogyne discolor Vogel \\
\hline & Peltogyne pauciflora Benth. \\
\hline & Peltophorum dubium Taub. \\
\hline & $\begin{array}{l}\text { Piptadenia gonoacantha J.F.Macbr. } \\
\text { Pityrocarpa moniliformis (Benth.) Luckow \& R.W.Jobson } \\
\text { Platycyamus regnellii Benth. }\end{array}$ \\
\hline & $\begin{array}{l}\text { Plathymenia reticulata Benth. } \\
\text { Poincianella pyramidalis (Tul.) L.P.Queiroz }\end{array}$ \\
\hline & $\begin{array}{l}\text { Pseudopiptadenia contorta (DC.) G.P.Lewis \& M.P.Lima } \\
\text { Pterogyne nitens Tul. }\end{array}$ \\
\hline
\end{tabular}




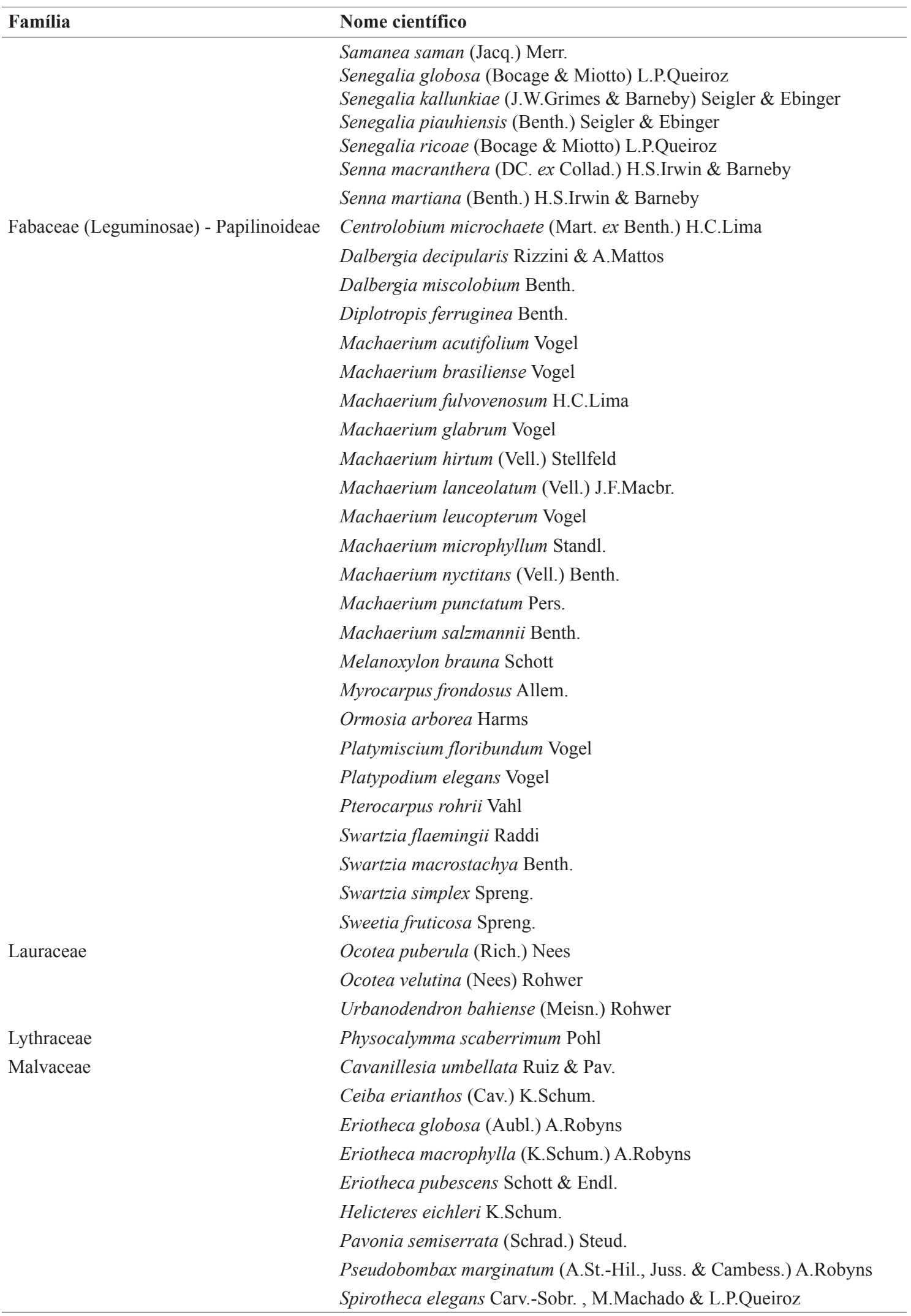




\begin{tabular}{|c|c|}
\hline Família & Nome científico \\
\hline \multirow[t]{5}{*}{ Meliaceae } & Guarea macrophylla Vahl \\
\hline & Trichilia elegans A.Juss. \\
\hline & Trichilia emarginata (Turcz.) C.DC. \\
\hline & Trichilia lepidota Mart. \\
\hline & Trichilia pseudostipularis C.DC. \\
\hline \multirow[t]{5}{*}{ Myrtaceae } & Eugenia ligustrina (Sw.) Willd. \\
\hline & Blepharocalyx salicifolius (Kunth) O.Berg \\
\hline & Eugenia punicifolia (Kunth) DC. \\
\hline & Myrcia splendens DC. \\
\hline & Myrcia tenuifolia (O.Berg) Sobral \\
\hline \multirow[t]{2}{*}{ Nyctaginaceae } & Andradea floribunda Allemão \\
\hline & Guapira opposita (Vell.) Reitz \\
\hline Olacaceae & Ximenia americana $\mathrm{L}$. \\
\hline Peraceae & Pogonophora schomburgkiana Miers ex Benth. \\
\hline Phyllanthaceae & Margaritaria nobilis L.f. \\
\hline Polygonaceae & Ruprechtia apetala Wedd. \\
\hline Primulaceae & Myrsine coriacea (Sw.) R.Br. \\
\hline Rhamnaceae & Rhamnus sphaerosperma Sw. var. pubescens (Reissek) M.C.Johnst. \\
\hline \multirow[t]{9}{*}{ Rubiaceae } & Alseis floribunda Schott \\
\hline & Amaioua guianensis Aubl. \\
\hline & Amaioua intermedia Mart. \\
\hline & Cordiera concolor (Cham.) Kuntze \\
\hline & Coutarea hexandra (Jacq.) K.Schum. \\
\hline & Ixora brevifolia Benth. \\
\hline & Ladenbergia hexandra Klotzsch \\
\hline & Randia $\operatorname{armata}(\mathrm{Sw}.) \mathrm{DC}$. \\
\hline & Tocoyena bullata Mart. \\
\hline \multirow[t]{6}{*}{ Rutaceae } & Esenbeckia febrifuga A.Juss. \\
\hline & Esenbeckia grandiflora Mart. \\
\hline & Metrodorea mollis Taub. \\
\hline & Pilocarpus spicatus A.St.-Hil. \\
\hline & Zanthoxylum fagara Sarg. \\
\hline & Zanthoxylum rhoifolium Lam. \\
\hline \multirow[t]{2}{*}{ Salicaceae } & Casearia guianensis (Aubl.) Urb. \\
\hline & Casearia sylvestris $\mathrm{Sw}$. \\
\hline \multirow[t]{5}{*}{ Sapindaceae } & Cupania oblongifolia Mart. \\
\hline & Cupania rubiginosa Radlk. \\
\hline & Diatenopteryx sorbifolia Radlk. \\
\hline & Dilodendron bipinnatum Radlk. \\
\hline & Matayba guianensis Aubl. \\
\hline \multirow[t]{3}{*}{ Sapotaceae } & Pouteria gardneri (Mart. \& Miq.) Baehni \\
\hline & Pradosia lactescens (Vell.) Radlk. \\
\hline & Sideroxylon obtusifolium (Roem. \& Schultes.) T.D.Penn. \\
\hline
\end{tabular}


Tabela 3 - Caracteres e estados de caracteres utilizados na chave de identificação.

Table 3 - Characters and character states used in the identification key

\begin{tabular}{|c|c|}
\hline Caractere & Estados de caractere \\
\hline Apresenta folhas verdadeiras & sim/não \\
\hline Filódios em formato de palma & sim/não \\
\hline Apresenta caule lenhoso & $\operatorname{sim} /$ não \\
\hline Tipo de filotaxia & alterna/oposta/verticilada \\
\hline Filotaxia Alterna & espiralada/dística/rosetada \\
\hline Limbo foliar & simples/composto \\
\hline Limbo foliar composto & digitado/pinado/bipinado/unifoliolado/bifoliolado/trifoliolado \\
\hline Característica da ráquis & alado/acanalado/zigue-zague/cilíndrico \\
\hline Número de jugas & $2-\mathrm{a} / 6-8 / 10-12 / 14-16 / 18-20 / 22-24 / 26-28 / 30$ ou mais \\
\hline Número de foliólulos & $4-8 / 9-12 / 13-16 / 17-20 / 21-30 / 31-40 /$ mais de 40 \\
\hline Folíolo ou juga terminal diferente dos demais & sim/não \\
\hline Limbo composto penado & imparipinado/paripinado \\
\hline Apresenta peciólulo & $\operatorname{sim} /$ não \\
\hline Número de folíolos imparipinados & 3/5-7/9-11/13-15/17-19/21-29/mais de 31 \\
\hline Número de folíolos paripinados & $2 / 4 / 6-8 / 10-14 / 16-18 / 20-28 /$ mais de 30 \\
\hline Com folíolo abortado ou pseudoabortado & $\operatorname{sim} /$ não \\
\hline Número de folíolos digitados & 4-6/6-7/mais de 7 \\
\hline Margem do limbo foliar & inteira/crenada/dentada/serrada/com espinhos \\
\hline Margem do limbo foliar inteira & lisa/revoluta/ondulada \\
\hline Forma do limbo & $\begin{array}{l}\text { elíptico/ovado/obovado/oblongo/lanceolado/orbicular/cordado/acicular/ } \\
\text { deltóide/em forma de faca/assimétrico }\end{array}$ \\
\hline Base do limbo & aguda/obtusa/truncada/assimétrica/cordada/decurrente/arredondada \\
\hline Ápice do limbo & $\begin{array}{l}\text { acuminado/acuminado e apiculado/acuminado e mucronado/agudo/ } \\
\text { agudo e apiculado/agudo e mucronado/arredondado/arredondado } \\
\text { e apiculado/arredondado e mucronado/emarginado/emarginado e } \\
\text { mucronado/obtuso/obtuso e mucronado/obtuso e apiculado }\end{array}$ \\
\hline Tipo de nervação & $\begin{array}{l}\text { broquidódroma/cladódroma/craspedódroma/campdódroma/ } \\
\text { eucampdódroma/acródroma/acródroma e broquidódroma/ } \\
\text { reticulódroma/uninérvea/sem nervação aparente }\end{array}$ \\
\hline Saliência de nervura & em ambas as faces/face abaxial/face adaxial/em nenhuma das faces \\
\hline $\begin{array}{l}\text { Saliência de nervura na face abaxial do limbo } \\
\text { foliar }\end{array}$ & $\begin{array}{l}\text { e nervuras adaxiais a nível do limbo/e nervuras adaxiais impressas/ } \\
\text { saliência abaxial apenas na nervura principal }\end{array}$ \\
\hline $\begin{array}{l}\text { Saliência de nervação em ambas as faces do } \\
\text { limbo foliar }\end{array}$ & de todas as nervuras/apenas das nervuras principais \\
\hline Apresenta nervura coletora & sim/não \\
\hline Limbo discolor & sim/não \\
\hline Discolor com face abaxial & verde mais claro/amarelado-alaranjado/esbranquiçado-prateado \\
\hline Apresenta pecíoloApresenta pecíolo & $\operatorname{sim} /$ não sim/não \\
\hline Característica do pecíolo & alado/acanalado/cilíndrico \\
\hline Folhas inseridas em braquioblástos & $\operatorname{sim} /$ não \\
\hline
\end{tabular}




\begin{tabular}{|c|c|}
\hline Caractere & Estados de caractere \\
\hline Apresenta glândula(s) & sim/não \\
\hline Tipo de glândula(s) & nectário extrafloral/translúcida (no limbo)/intumescimento/outras \\
\hline Posição da(s) glândula(s) & $\begin{array}{l}\text { todo o limbo/margem do limbo/base do limbo/pecíolo/raquíola/ápice do } \\
\text { raque/ápice do pecíolo/meio do pecíolo/entre jugas no raque }\end{array}$ \\
\hline Glândula(s) em todo limbo & $\begin{array}{l}\text { translúcidas e circulares/traslúcidas e em forma de bastonetes/circulares } \\
\text { de cor mais clara na face abaxial }\end{array}$ \\
\hline Apresenta estípula & sim/não \\
\hline Local da estípula & intrapeciolar/interpeciolar/ao lado da inserção do pecíolo/na gema apical \\
\hline Tipo de estípula & ramento/lanceolar/piramidal/estipelas/apenas marca \\
\hline Oxidação das folhas & sim/não \\
\hline Folha no ápice dos ramos & sim/não \\
\hline Presença de ócrea & sim/não \\
\hline Consistência do limbo & membranacea/cartácea/coreácea \\
\hline Apresenta lenticelas & sim/não \\
\hline Local das lenticelas & pecíolo/ramos \\
\hline Apresenta pubescência & sim/não \\
\hline Tipo de pubescência & pequenos pelos/cínero/ferrugíneo/cílios/tufos/puberulenta/velutina \\
\hline Local de pubescência & $\begin{array}{l}\text { face abaxial do limbo/face adaxial do limbo/ambas as faces do limbo/ } \\
\text { nervuras/nervuras principal adaxial/nervura principal abaxial/ráquis- } \\
\text { raquíola/pecíolo-peciolulo/ramo/gema }\end{array}$ \\
\hline Presença de pulvino & sim/não \\
\hline Presença de odor característico & sim/não \\
\hline Presença de espinho & sim/não \\
\hline Quantidade de espinhos & um por nó/dois por nó/múltiplo \\
\hline Presença de acúleos & sim/não \\
\hline Tipo de acúleo & reto/curvado \\
\hline Local do acúleo & ramos/raque/folha \\
\hline Apresenta algum tipo de exsudato & sim/não \\
\hline Tipo de exsudato & látex/outro \\
\hline Apresenta embira & $\operatorname{sim} /$ não \\
\hline Apresenta estrias nos ramos & sim/não \\
\hline Apresenta ramos caniculados & sim/não \\
\hline Apresenta ramos suberosos & $\operatorname{sim} /$ não \\
\hline $\begin{array}{l}\text { Apresenta ramo de cor diferente do pecíolo ou } \\
\text { pecíolo mais raque }\end{array}$ & sim/não \\
\hline Ramo em zigue-zague & sim/não \\
\hline Ramos com cristas & sim/não \\
\hline Forma do ramo & achatado/angular/cilíndrico \\
\hline Ramo oxidado & sim/não \\
\hline Ramo avermelhado & sim/não \\
\hline Apresenta nós muito evidentes & sim/não \\
\hline
\end{tabular}


al. (2009), Couto et al. (2011) e Alves et al. (2015). As famílias Malvaceae e Rubiaceae apresentaram nove espécies cada, Euphorbiaceae (oito), Anacardiaceae (sete), Rutaceae e Boraginaceae (seis), o gênero mais frequente foi Machaerium (Leguminosae) com 11 espécies.

A base de dados "Árvores das Florestas Estacionais da Bahia" foi armazenada online na plataforma do $\mathrm{Xper}^{3}$, permitindo a utilização de forma livre e está disponível em (<http:// www.xper3.fr/xper3GeneratedFiles/publish/ identification/337734661618352647/mkey. html $>$ ).

Na plataforma, o "layout" permite visualizar os caracteres e os seus estados do lado esquerdo, enquanto a lista de espécies aparece do lado direito (Fig. 4) conforme a seleção de caracteres. Os estados apresentaram coloração que vai do verde ao branco, quanto maior a intensidade da cor verde, menor quantidade de espécies que apresentam aquele caractere. Assim, à medida que se seleciona os estados dos caracteres, vão sendo eliminados as espécies que não apresentaram caracteres escolhidos durante o processo de identificação, até restar apenas uma espécie (táxon identificado perante as escolhas).

Desta forma, por ser uma chave interativa de identificação, se torna também intuitiva a sua utilização. Este tipo de ferramenta pode ser utilizado tanto na pesquisa científica, quanto como ferramenta de ensino nas aulas de morfologia, taxonomia vegetal e dendrologia. Também, pode se tornar uma ferramenta para identificação de espécies para licenciamentos e outras áreas que

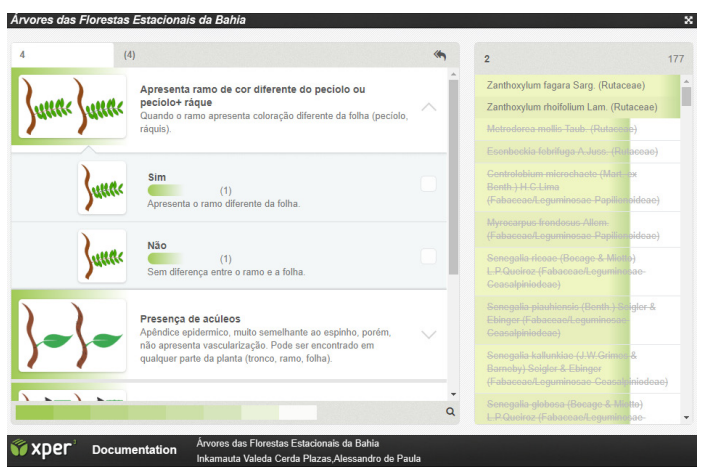

Figura 4 - Tela da área de identificação, mostrando os atributos com as images ilustrativas, e na lateral direita, a lista de espécies.

Figure 4 - Screen of the identification place, showing the attributes with illustrative images, and on the lateral side, the list of species. dependem diretamente da determinação dos táxons vegetais.

A chave pode apresentar erro caso a espécie a ser identificada apresente-se morfologicamente muito diferente das analisadas. Isto pode ocorrer com algumas espécies por se apresentarem em localidade com condições edafoclimáticas e de sítios diferentes. Além disso, espécies de ampla ocorrência, apresentam geralmente grande variação morfológica, dificultando sua identificação. No entanto, a chave de múltiplas entradas (interativa) permite incluir vários estados de caráter ao mesmo tempo diminuindo assim, erros de identificação.

A maioria das espécies apresentadas nos estudos de Funch et al. (2008) e Couto et al. (2011) para as Florestas Estacionais da Bahia, se encontram nesta chave. Além disso, devido à similaridade florística entre as Florestas Estacionais, esta chave pode ser aplicada na identificação de espécies de Floresta Estacionais de outros estados (Bittrich et al. 2012; ChrétiennotDinet et al. 2014; Couto et al. 2011).

\section{Agradecimentos}

Os autores agradecem à FAPESB - Fundação de Amparo à Pesquisa do Estado da Bahia, a bolsa de Iniciação científica (2016-2017) concedida ao primeiro autor; ao Herbário da Universidade Estadual de Feira de Santana, em especial ao curador Luciano Paganucci de Queiroz; e aos revisores deste trabalho, ao serem tão cuidadosos e preocupados com a qualidade do trabalho.

\section{Referências}

APG IV - Angiosperm Phylogeny Group (2016) Angiosperm Phylogeny Website. Disponível em <http://www.mobot.org/MOBOT/research/ APweb/>. Acesso em 30 julho 2018.

Alves M, Oliveira RB, Teixeira RS, Guedes LMS \& Roque N (2015) Levantamento florístico de um remanescente de Mata Atlântica no litoral norte do estado da Bahia, Brasil. Hoehnea 42: 581-595. DOI: 10.1590/2236-8906-06/2015

Bittrich V, Souza CSD, Coelho RLG, Martins MV, Hopkins MJG \& Amaral MCE (2012) An interactive key (Lucid) for the identifying of the genera of seed plants from the Ducke Reserve, Manaus, AM, Brazil. Rodriguésia 63: 055-064. DOI: 10.1590/ S2175-78602012000100005

BFG - The Brazil Flora Group (2018) Brazilian Flora 2020: innovation and collaboration to meet Target 1 of the Global Strategy for Plant Conservation (GSPC). Rodriguésia 69: 1513-1527. 
Bradshaw AD (1965) Evolutionary significance of phenotypic plasticity in plants. Advances in Genetics 13: 115-155. DOI: 10.1016/S00652660(08)60048-6

Campanili M \& Prochnow M (2006) Mata Atlântica: uma rede pela floresta. Ed. RMA, Brasília. 332p.

Cardoso DBOS, França F, Novais JS, Ferreira MHS, Santos RM, Carneiro VMS \& Gonçalves JM (2009) Composição florística e análise fitogeográfica de uma floresta semidecídua na Bahia, Brasil. Rodriguésia 60: 1055-1076. DOI: 10.1590/21757860200960416

Carmo JAM \& Simões AO (2017) Rubiaceae no município de Camanducaia, Serra da Mantiqueira, Minas Gerais: sinopse e chave interativa. Rodriguésia 68: 1411-1429. DOI: 10.1590/21757860201768421

Chrétiennot-Dinet M, Desreumaux N \& VignesLebbe R (2014) An interactive key to the Chrysochromulina species (Haptophyta) described in the literature. PhytoKeys 34: 47-60. DOI: 10.3897/phytokeys.34.6242

Couto APL, Funch LS \& Conceição AA (2011) Composição florística e fisionomia de floresta estacional semidecídua submontana na Chapada Diamantina, Bahia, Brasil. Rodriguésia 61: 391-405. DOI: $10.1590 / 2175-7860201162213$

Eltink M, Ramos E, Torres RB, Tamashiro JY, Galembeck E \& Kimura E (2011) Chave de identificação de espécies do estrato arbóreo da Mata Atlântica em Ubatuba (SP), com base em caracteres vegetativos. Biota Neotropica 11: 393-405. DOI: 10.1590/ S1676-06032011000200037

Font Quer P (2001) Diccionario de batánica. Ed. Labor, Barcelona. 1280p.

Funch LS, Rodal MJN \& Funch RR (2008) Floristic aspects of the forests of the Chapada Diamantina, Bahia, Brazil. In: Thomas W \& Briton EG (org.) The Atlantic Coastal Forest of Northeastern Brazil. Memoirs of the New York Botanical Garden Press 100: 193-220.
Gonçalves EG \& Lorenzi H (2007) Morfologia vegetal: organografia e dicionário ilustrado de morfologia de plantas vasculares. Ed. Instituto Plantarum de Estudos da Flora, São Paulo. 512p.

IBGE - Instituto Brasileiro de Geografia e Estatística (2012) Manual técnico da vegetação brasileira. $2^{a}$ ed. ED. IBGE, Rio de Janeiro. 271p.

Judd WS, Campbell CS, Kellogg EA, Stevens PF \& Donoghue MJ (2009) Sistemática vegetal: um enfoque filogenético. $3^{\text {a }}$ ed. Ed. Armed, Porto Alegre. 632 p.

Marchiori JNC (2004) Elementos de dendrologia. Ed. UFSM, Santa Maria. 176p.

Pinheiro AL \& Almeida EC (2008) Fundamentos de taxonomia e dendrologia tropical: introdução aos estudos dendrológicos. Vol. 1. Ed. UFV, Viçosa - Departamento de Engenharia Florestal e Departamento de Biologia Vegetal. 72p.

Ramalho RS (1978) Dendrologia: terminologia. Vol. 1. Ed. UFV, Viçosa. 123p.

Roderjan CV (1983) Morfologia do estágio juvenil de 24 espécies arbóreas de uma floresta com araucária. Dissertação de Mestrado. Universidade Federal do Paraná, Curitiba. 148p.

Santo FSE, Siqueira AA \& Rapini A (2013) Chave interativa para a identificação das espécies da Aliança Tabebuia (Bignoniaceae) no estado da Bahia, Brasil. Biota Neotropica 13: 344-349. DOI: 10.1590/S1676-06032013000300034

Saueressig D, Saueressing A \& Inoue MT (2009) SIDOL - Sistema de identificação dendrológica on-lin. Ambiência - revista do Setor de Ciências Agrárias e Ambientais 5: 117-133.

Soares Filho AO (2012) Fitogeografia e estrutura das florestas estacionais deciduais no Brasil. Tese de Doutorado. Universidade Estadual de Feira de Santana, Feira de Santana. 346p.

SOS Mata Atlântica \& INPE (2019) Atlas dos remanescentes florestais da Mata Atlântica. Relatório técnico - período 2017-2018. Ed. INPE, São Paulo. 68p. 